TIPA. Travaux interdisciplinaires sur la parole et le langage

$36 \mid 2020$

Comment le corps coconstruit les discours et le sens

\title{
Utilisation du geste illustratif par les apprenants coréens dans la classe de langue
}

Use of illustrative gesture by Korean learners in the language class

Shin-Tae Kang

\section{OpenEdition}

1 Journals

Édition électronique

URL : http://journals.openedition.org/tipa/3793

DOI : $10.4000 /$ tipa.3793

ISSN : 2264-7082

Éditeur

Laboratoire Parole et Langage

Référence électronique

Shin-Tae Kang, «Utilisation du geste illustratif par les apprenants coréens dans la classe de langue », TIPA. Travaux interdisciplinaires sur la parole et le langage [En ligne], 36 | 2020, mis en ligne le 23 septembre 2020, consulté le 27 janvier 2021. URL : http://journals.openedition.org/tipa/3793 ; DOI : https://doi.org/10.4000/tipa.3793

Ce document a été généré automatiquement le 27 janvier 2021.

La revue TIPA. Travaux interdisciplinaires sur la parole et le langage est mise à disposition selon les termes de la licence Creative Commons Attribution - Pas d'Utilisation Commerciale - Pas de Modification 4.0 International. 


\title{
Utilisation du geste illustratif par les apprenants coréens dans la classe de langue
}

\author{
Use of illustrative gesture by Korean learners in the language class
}

\author{
Shin-Tae Kang
}

\section{Introduction}

1 Lors d'une conversation, un locuteur accompagne souvent son discours de gestes et de mimiques pour, notamment, aider à faire se faire comprendre de ses partenaires. Selon Kendon (2004: 360), le déploiement réciproque de la parole et du geste dans l'énonciation est le résultat d'intentions communicatives des orateurs. Ce langage gestuel peut s'inscrire dans le rapport que le corps humain établit avec son environnement au sein d'un espace culturel donné. Colletta stipule que la langue ne se présente jamais nue mais au contraire toujours habillée du costume de la voix du locuteur et du pardessus de ses attitudes, gestes, mimiques et regards (2005: 1). Ainsi notre gestuelle, notre façon de parler et d'établir un contact visuel sont autant de moyens de communication non verbaux et paraverbaux qui influencent les messages que nous transmettons verbalement. Colletta (2011) estime que les messages transmis par les canaux visuels (statiques et kinésiques) viennent en complément de ceux qui sont émis par le canal auditif lors d'un acte de communication langagier, aussi bien en production qu'en réception. Les messages qui transitent par les canaux visuels constituent une composante visuelle du langage qui est présente partout, non seulement dans la vie quotidienne mais également sur des terrains spécifiques comme les situations où deux langues se côtoient. C'est ainsi qu'en classe de langue, l'enseignant et les apprenants utilisent aussi leur corps pour communiquer et pour faciliter la compréhension et l'expression d'une langue étrangère dans les séquences d'échange (Calbris et Porcher, 1989), l'enseignant se servant plus spécifiquement des gestes pour animer une classe, informer et évaluer les apprenants (Tellier, 2008; Azaoui, 2014a). Il est donc judicieux de voir comment le corps des 
participants à l'échange coconstruit le discours et le sens dans cette situation propice aux malentendus et incompréhensions.

L'objectif de cette contribution est d'identifier et analyser plus particulièrement les gestes utilisés par les apprenants coréens face à leur enseignant français dans le cadre de cours de français langue étrangère à l'université en Corée du Sud. Pour cette analyse de pratiques d'apprenants, nous utiliserons la catégorisation fonctionnelle du geste établie par Cosnier $(1982,1997)$ dans laquelle nous retiendrons les gestes communicatifs coverbaux illustratifs. Nous évoquerons par ailleurs les dimensions définies par McNeill $(1992,2005)$ à qui nous emprunterons le terme de geste métaphorique. Nous nous pencherons donc essentiellement sur ces nombreux gestes illustratifs, qu'ils soient déictiques, iconiques ou métaphoriques et révélateurs des particularités discursives et culturelles des apprenants coréens dans leurs interactions avec les enseignants français. Nous resterons attentifs à la séquentialisation des gestes et de la parole au sein de l'interaction, en portant une attention particulière à la prépondérance que peut prendre le geste sur la parole dans les situations de lacunes langagières propres à notre terrain d'étude, à savoir la classe de langue étrangère avec des enseignants qui ne connaissent pas, ou très peu, la langue maternelle des apprenants. En effet nous faisons l'hypothèse que, dans ce contexte précis, lorsque la langue en acquisition est défaillante, ce n'est pas la langue maternelle qui prend le relais mais plutôt le langage gestuel. Pour cela nous analyserons à chaque fois la réaction de l'enseignant afin de voir si l'intention de l'apprenant a été comprise et la façon dont il a géré l'échange. Dans le cadre conceptuel nous développerons les notions évoquées dans cette introduction. Puis nous présenterons la méthodologie suivie et le corpus que nous avons créé pour cette étude. Enfin nous mènerons notre analyse qualitative sur les gestes illustratifs des apprenants en tentant d'expliquer dans quels contextes ils sont exécutés.

\section{Cadre conceptuel}

Il n'existe pas de typologie universelle des gestes car la volonté d'établir un schéma d'annotation exhaustif et commun à tous les chercheurs se heurte à sa mise en pratique, l'utilisation de telle ou telle typologie étant principalement sous-tendue par l'objectif de recherche (Calbris, 2011: 102). Nous retiendrons pour notre étude les théories de McNeill $(1992,2005)$ et de Cosnier $(1982,1997)$ car leurs classifications fonctionnelles permettent de fournir des informations qualitatives sur l'interaction en face à face. Traverso (1999: 15) rappelle la notion de multicanalité dans la communication en évoquant les canaux auditif et visuel qui entrent tous deux dans la composition du message ainsi que dans l'engagement des participants. Par le canal auditif transitent le verbal, qui correspond à ce qui est énoncé par les interactants, et le paraverbal, qui est indissolublement lié au verbal et qui correspond à la prosodie et aux autres manifestations de la voix. Par le canal visuel transite le non verbal qui correspond aux activités mimo-posturo-gestuelles et dans lesquelles se situent les coverbaux qui accompagnent la production verbale, par exemple pour l'illustrer (Traverso, 1999 : 16) et qui sont l'objet de notre contribution. Cosnier voit dans l'interaction en face à face la synergie de deux voies concomitantes : l'une discursive par laquelle est acheminé l'aspect signifiant ou informatif de l'énoncé ou encore le contenu propositionnel, et l'autre pragmatique qui en assure la maintenance et la régulation [...] (1997 : 2). La voie discursive - 
celle qui assure la composition du message - est évidemment principalement assurée par le verbal, mais le sens peut aussi transiter par une activité mimo-posturo-gestuelle, ce qui conduit Cosnier à introduire le terme de gestualité discursive (1997 : 2). De même, la voie pragmatique - celle qui marque l'engagement des participants - nécessite autant le verbal et le paraverbal que les gestes de régulation. Ceci amène Cosnier à la constatation suivante : Le postulat de multicanalité nous oblige [...] à ne pas sélectionner a priori les aspects verbaux ou non verbaux mais à les considérer comme synergiques. (1997:2). Nous positionnons notre recherche dans cette perspective qui envisage la parole verbale et le geste coverbal comme deux modalités fonctionnant ensemble pour former le discours oral (Colletta, $2004: 104$ ).

4 Ces différentes voies empruntées par les gestes dans l'interaction sont à la base de la catégorisation fonctionnelle proposée par Cosnier (1982, 1997). Il distingue deux grandes catégories : d'une part les gestes communicatifs et d'autre part les gestes dits extra-communicatifs. Les gestes communicatifs ont pour but de transmettre un message, ils sont souvent volontaires et explicites, et participent directement à l'interaction. Cosnier divise les gestes communicatifs en quatre catégories : les quasilinguistiques appelés aussi 'emblèmes' (qui peuvent se substituer à un mot ou une phrase et qui sont marqués par la culture du locuteur), les coverbaux (qui accompagnent la parole), les synchronisateurs (qui servent à réguler des échanges dans l'interaction), et les phonogènes (mouvements des lèvres indispensables au langage parlé). Les gestes coverbaux n'ont pas de sens conventionnel et ont besoin de la parole pour créer du sens; on les classe en gestes paraverbaux proprement dits, liés aux traits phonétiques et syntaxiques (gestes de battement comme les mouvements de la tête et des mains pour souligner l'intonation ou l'emphase...), expressifs, pour accompagner l'expression de sentiments-sensations (joie, colère, tristesse...) et illustratifs. Les gestes illustratifs sont caractérisés par le fait qu'ils font référence à quelqu'un, quelque chose ou une idée qu'ils illustrent. Par conséquent ils peuvent avoir valeur déictique (gestes de pointage qui désignent un référent de la parole, comme montrer du doigt l'objet dont on parle), valeur iconique (représentation par le mime d'un concept concret que Cosnier différencie en spatiographique pour la disposition spatiale, pictographiques pour la forme et kinémimique pour l'action) ou encore valeur métaphorique (représentation d'un concept abstrait et que Cosnier appelle idéographique). Ce découpage des gestes coverbaux se retrouve dans les dimensions de McNeill (1992, 2005) qui distingue le geste déictique (geste de pointage), iconique (geste illustratif d'un concept concret), métaphorique (geste illustratif d'un concept abstrait) et de battement (geste rythmant la parole, sans contenu sémantique). Pavelin (2002: 39) précise la coverbalité du geste iconique par le fait que dans les gestes iconiques, les mains fonctionnent en tant que symboles qui représentent par leur forme et par leur façon de se mouvoir le contenu qui se rapporte au contenu linguistique co-occurrent.

5 Par ailleurs, certains spécialistes (Calbris et Porcher, 1989) ont souligné l'importance du geste en didactique des langues. Il ne faut pas seulement le percevoir comme un objet externe, mais aussi l'interpréter comme intention énonciative, expression propre du gesticulateur, et élément d'une situation globale (1989: 38). Tellier a introduit la notion de geste pédagogique, qui a pour objectif premier de faciliter l'accès au sens en langue étrangère. Il agit comme une traduction gestuelle des paroles de l'enseignant. Le lien entre le geste et la parole qu'il accompagne est donc crucial (2008: 43). D'ailleurs le geste pédagogique est essentiellement un geste coverbal (déictique, iconique et 
métaphorique principalement) avec la prépondérance des mimes, même si le locuteur peut avoir recourt aux emblèmes pédagogiques pour des raisons communicationnelle ou contextuelle dans le cas d'une évaluation positive en classe de FLS par exemple (Azaoui, 2014b). De plus Tellier fait le constat qu'un geste pédagogique, est souvent élaboré à partir de traits caractéristiques du référent (2008: 47) afin d'être facilement identifiable par l'interlocuteur.

Des études montrent que les apprenants de langue seconde utilisent davantage le geste lorsqu'ils parlent leur L2 que lorsqu'ils parlent leur langue maternelle (Gullberg, 1998 ; Stam, 2008). Les apprenants de langue seconde sont susceptibles d'utiliser des gestes illustratifs comme moyen de se reporter consciemment à des éléments lexicaux lorsque le sens des mots n'est pas clair, afin de faciliter la compréhension de l'interlocuteur (McCafferty \& Ahmed, 2000). Gullberg a notamment étudié les gestes de suédois (1998) et néerlandophones (2005) apprenant le FLE en contexte exolingue. Elle montre que les apprenants d'une langue étrangère ont une véritable stratégie de communication gestuelle qui va bien au-delà de la simple utilisation de gestes compensatoires principalement mimétiques remplaçant la parole. Elle constate que les gestes iconiques et certains gestes déictiques (ceux de pointage concret) sont utilisés par les apprenants pour résoudre des problèmes de lexique en incitant leur interlocuteur à participer à l'élaboration du sens dans une démarche d'élicitation: These gestures are often used to elicit lexical help from the interlocutor (1998: 229). Quant aux gestes déictiques abstraits, les apprenants les utiliseraient pour surmonter des difficultés grammaticales, par exemple pour désambiguïser (2005) ce qu'ils ne distinguent pas à l'oral (l'ambiguïté d'un même syntagme nominal surexploité est levée par des gestes anaphoriques différenciés). Enfin, les apprenants utiliseraient des gestes pour signaler métalinguistiquement la présence d'un problème tel qu'une recherche lexicale.

\section{Méthodologie et corpus d'analyse}

7 Notre analyse est fondée sur la description de l'activité d'enseignement/apprentissage du français langue étrangère (désormais FLE) dans la classe de langue entre enseignants français et apprenants coréens. La recherche descriptive a pour but de décrire des faits, des objets, des événements, des comportements en utilisant différentes stratégies d'observation mais a aussi pour visée complémentaire de comprendre ou d'expliquer (Gagné et al., 1989: 39). Pour cela il est indispensable de recueillir des données empiriques et authentiques dans un cadre écologique qui a vocation à être employé pour désigner des situations pédagogiques non créées pour les besoins d'une étude (Dejean-Thircuir \& Mangenot, 2006 : 9). C'est dans ce cadre que l'on trouve des situations "naturelles » (Traverso, 2008) dans le sens d'un corpus non construit artificiellement par le chercheur.

Nous nous sommes donc attachés à décrire et analyser la gestualité des apprenants coréens dans l'interaction pédagogique entre enseignants français natifs et apprenants coréens. Nous avons créé pour notre analyse un corpus d'enregistrements audio-vidéo de cours de FLE pour apprenants coréens. La plus grande majorité des enregistrements s'est déroulée lors de cours de conversation hebdomadaires dans des universités en Corée du Sud en début du second semestre. Ce corpus peut se résumer en quelques chiffres : captation de 5 cours de FLE, interactions entre 4 enseignants et 48 apprenants au sein de deux universités coréennes et une française, près de sept heures d'enregistrement. Après visionnage de l'ensemble de ces rushs nous avons isolé puis 
transcrit, selon la convention ICOR 2013, les passages remarquables comportant des gestes. Dans les transcriptions et les analyses, les enseignants sont désignés de façon générale par la lettre $\mathrm{P}$ ou alors ils sont identifiés par les lettres $\mathrm{A}, \mathrm{B}, \mathrm{C}$ ou $\mathrm{D}$; les apprenants sont désignés par la lettre $\mathrm{E}$ suivie d'un indice détaillé dans la convention de transcription. Des captures d'écran viennent appuyer les commentaires afin d'illustrer les gestes des interactants.

9 La licence en Corée se déroule sur quatre ans. Nous avons enregistré des cours de licence de 2ème année qui correspondent en moyenne au niveau A2/B1 et des cours de 3ème année qui correspondent en moyenne au niveau B1/B2. Nous présentons dans ce qui suit les profils des participants, enseignants français et apprenants coréens :

- l'enseignante A est étudiante en Master 2 de FLE en France et a fait son stage de 8 mois en Corée à l'université nationale de Chungbuk à Cheongju; sa classe compte 6 étudiants de 2ème année ;

- l'enseignant B exerce à l'université nationale de Séoul et a une expérience de 5 ans dans ce pays ; sa première classe compte 7 étudiants de 3ème année ; sa seconde classe compte 16 étudiants de 2ème année ;

- l'enseignant C exerce à l'université nationale de Chungbuk à Cheongju depuis 6 ans ; sa classe compte 5 étudiants de 3ème année ;

- l'enseignant D exerce à l'université Lumière Lyon 2 ; ce passage est extrait d'un corpus adjacent enregistré à Lyon et a été retenu car il relate l'interaction entre une apprenante coréenne et l'enseignant de FLE; les 14 apprenants de ce cours sont de nationalités différentes et ont un niveau B1.

Ces quatre enseignants donnent leur cours de conversation intégralement en français et nous ont confié ne pas parler coréen. Cependant, pour ceux justifiant d'une présence en Corée de plus de 5 ans, nous pouvons penser qu'ils ont acquis quelques rudiments en coréen. Nous verrons si cela a une incidence sur les stratégies de compensation mises en œuvre par les apprenants coréens et sur la réaction des enseignants.

11 Le profil des apprenants est relativement homogène car ils ont presque tous le même âge (22 ans en moyenne), le même statut (tous étudiants universitaires en Corée) et sont répartis en classe de niveau (2ème ou 3ème année de licence). Nous savons que seulement quelques étudiants ont effectué un apprentissage du français en France, par contre nous ne savons pas si les autres étudiants ont suivi ou suivent des cours supplémentaires de soutien en FLE.

\section{Analyse qualitative}

12 Nous cherchons à savoir comment les apprenants coréens utilisent les gestes pour coconstruire leur discours et le sens dans la classe de FLE en Corée. Nous nous placerons tout d'abord dans les deux contextes suivants : lorsqu'ils doivent confirmer à leur enseignant la bonne compréhension d'un mot français et lorsqu'ils tentent de faire comprendre à leur enseignant le sens d'un mot dont ils ne connaissent pas la traduction en français. Puis nous analyserons des cas où les gestes servent à exprimer plus qu'un lexique. 


\section{4-1. Gestes de vérification lexicale}

Les cours de conversation se déroulent uniquement en langue cible en suivant la méthode directe. Comme il n'est pas fait usage de la méthode grammaire-traduction, il n'est pas toujours aisé pour l'enseignant de savoir si un mot de vocabulaire a été compris par les apprenants et c'est pour cela qu'il peut leur demander de l'expliquer ; c'est aussi une méthode pour animer le cours et faire participer les apprenants. Quelles réponses les apprenants peuvent-ils apporter : donner une définition du mot en livrant une phrase explicative ou bien trouver un synonyme? Il semble que les apprenants coréens exploitent la multicanalité de la communication en associant le geste à la parole. Nous nommerons les gestes employés par les apprenants dans ce contexte précis "gestes de vérification lexicale" car ils visent par leur vertu illustrative à vérifier leur bonne compréhension du sens d'un mot ou d'une idée émise par l'enseignant.

\section{Exemple 1 :}

Cet extrait se situe pendant un cours de français de niveau A2 dans une université nationale en Corée du Sud entre l'enseignante A et les apprenants coréens.

Transcription $1:$ « Féliciter »

\begin{tabular}{|l|l|l|}
\hline$(1)$ & $P$ & féliciter::: (02') qu'est-ce que ça veut dire féliciter \\
\hline$(2)$ & E1/E2 & (applaudissements : Image 1) \\
\hline$(3)$ & P & waouh (regarde les deux étudiantes) (rire) \\
\hline$(4)$ & E1/E2 & (éclats de rire) \\
\hline (5) & P & bravo/ allez::: féliciter \\
\hline
\end{tabular}

Image 1

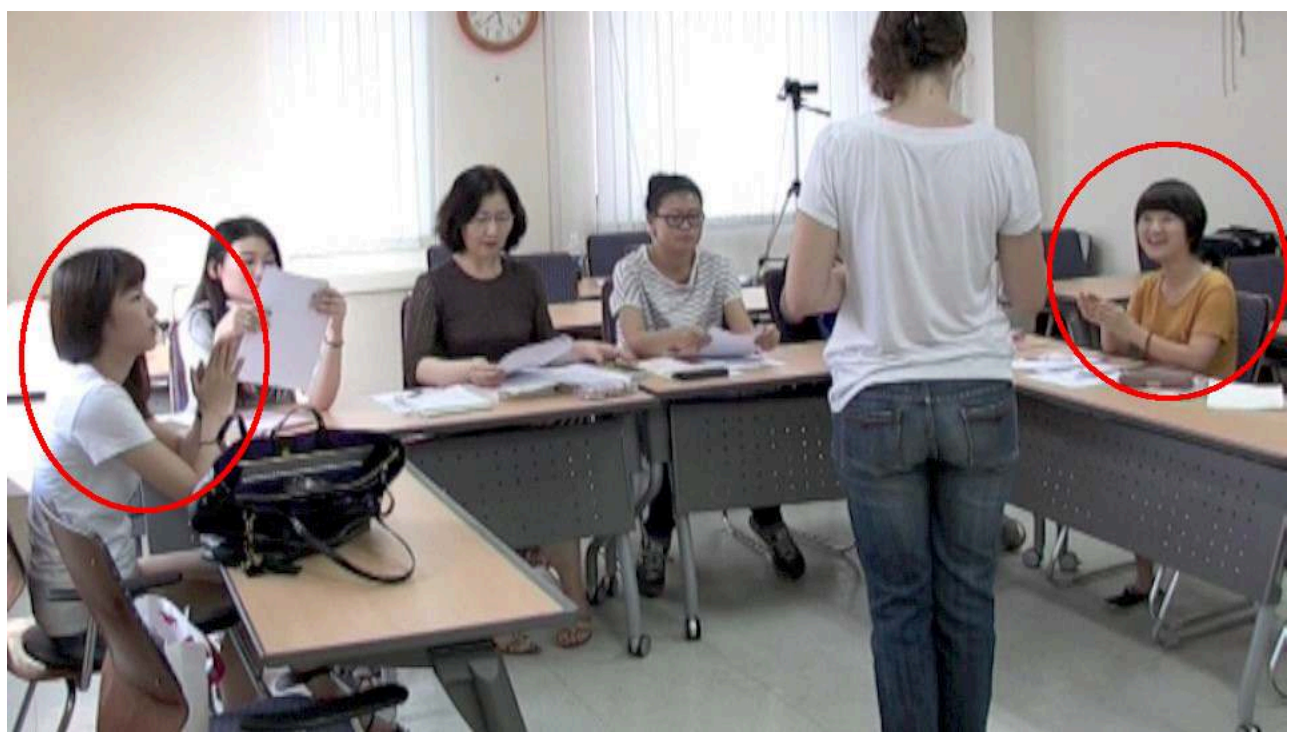

E1 et E2 applaudissent. 

veut dire féliciter?». Elle semble estimer que le verbe 'féliciter' est difficile à comprendre et réfléchit à la façon dont elle va expliquer ce verbe comme le laisse supposer la pause (02') de la ligne 1 « féliciter::: (02’) ».

Il est intéressant, dans la suite de l'interaction, de suivre les regards des deux apprenantes (E1 et E2) dirigés vers l'enseignante alors que les autres apprenantes ne répondent pas à la question et se concentrent sur le document. On peut interpréter cette attitude par le fait que les deux apprenantes connaissent le sens du verbe 'féliciter' et souhaitent le signifier à l'enseignante en la fixant du regard, une d'entre elles esquissant même un imperceptible mouvement de tête de haut en bas, comme un acquiescement. Elles livrent ensuite leur réponse en utilisant le même geste: elles applaudissent tout en regardant l'enseignante (Image 1). L'enseignante se retourne alors vers l'une et l'autre en les désignant du doigt, empruntant ainsi le même canal de communication, le canal visuel. Simultanément elle s'exclame « waouh » et toute la classe rit grâce au geste de vérification lexicale des deux apprenantes E1 et E2.

Puis, reprenant son rôle didactique, l'enseignante livre la transposition verbale de ce geste «bravo » et le terme initial « féliciter » qui est maintenant compris par toute la classe.

Ce geste de vérification lexicale des deux étudiantes est un geste emblème qui vise à donner le sens du verbe féliciter en le remplaçant par le verbe applaudir. Il a ici les fonctions de vérifier et signifier la compréhension du mot féliciter.

\section{Exemple 2 :}

Observons maintenant la deuxième transcription avec l'enseignant $B$ et un geste de vérification lexicale exécuté par une apprenante.

Transcription 2 : «Pigeon »

\begin{tabular}{|l|l|l|}
\hline$(1)$ & P & heu:::des:::animaux que vous n'aimez VRAIMENT pas/ (gestes) \\
\hline$(2)$ & $(02 ')$ & \\
\hline$(3)$ & ES & XXX (discutent en coréen) \\
\hline$(4)$ & E1 & Pi Pijou/ \\
\hline$(5)$ & P & BISOU/ \\
\hline$(6)$ & E1 & pi:::pi:::PIjon/ (regarde une camarade à côté) \\
\hline$(7)$ & P & AH PIGEON/ PIgeon/ d'accord PIGEON \\
\hline$(8)$ & E2 & qu'est-ce que c'est/ \\
\hline$(9)$ & E3 & je déteste celui XXX \\
\hline$(10)$ & E4 & (gestes avec les deux mains en regardant E2) \\
\hline$(11)$ & E5 & [c'est pas::: c'est pas un insecte hein:::/ (rire) \\
\hline
\end{tabular}




\begin{tabular}{|l|l|l|}
\hline$(12)$ & P & {$[$ heu::: (rire) $=$} \\
\hline$(13)$ & E3 & =bidoulki' / (parle en coréen) \\
\hline$(14)$ & P & qu'est-ce que c'est un pigeon/ vous pouvez nous dire/ \\
\hline$(15)$ & & (mêmes gestes que E4 avec les deux mains : Image 2) \\
\hline$(16)$ & E4 & (mêmes gestes avec les deux mains : Image 2) \\
\hline$(17)$ & E2 & on a XXX \\
\hline$(18)$ & P & oui c'est comme un aigle mais petit (geste 'petit') \\
\hline$(19)$ & E6 & mais::: \\
\hline$(20)$ & E2 & XXX \\
\hline$(21)$ & P & oui::: il y a beaucoup de pigeons à Paris par exemple hein/ \\
\hline$(22)$ & & il y en a un peu moins à Séoul mais heu il y en a \\
\hline$(23)$ & & quand même pas mal XXX \\
\hline$(24)$ & E6 & il y a XXX \\
\hline
\end{tabular}

Image 2

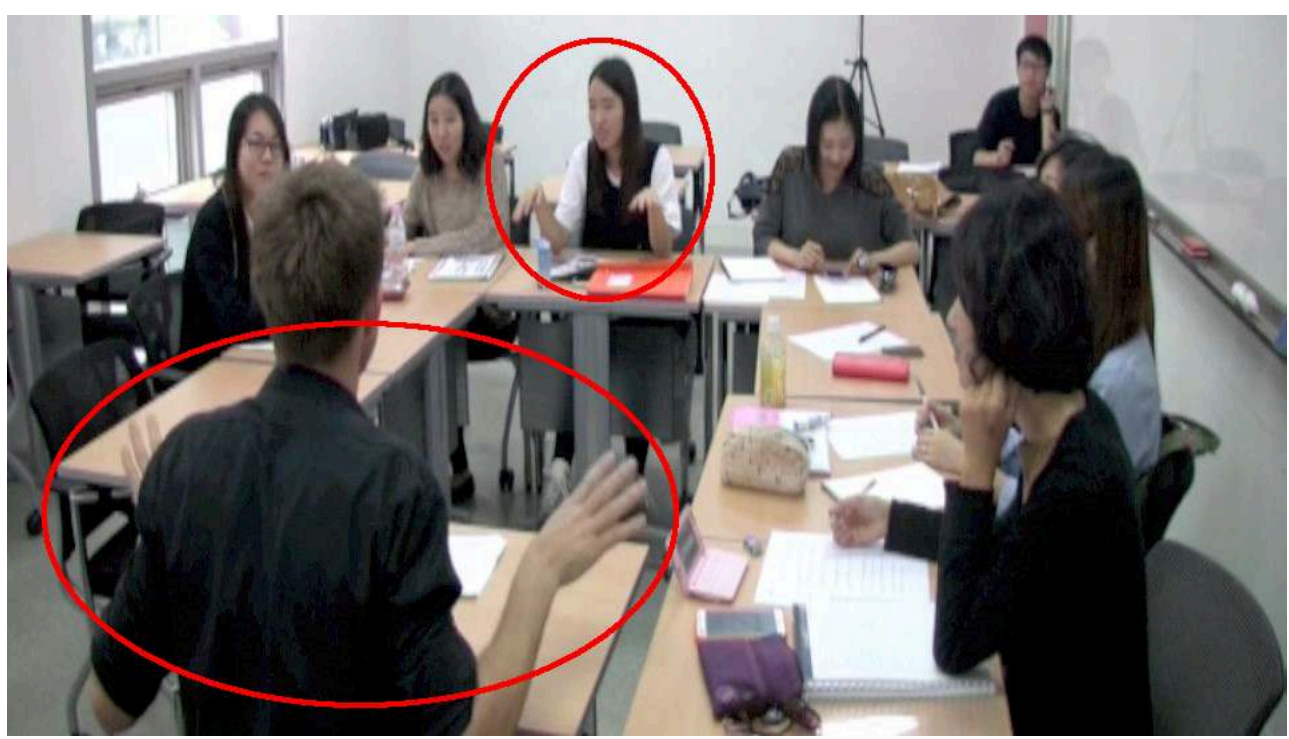

«Qu'est-ce que c'est un pigeon ? Vous pouvez nous dire?»

Dans cette transcription, l'enseignant B demande aux apprenants de citer le nom d'un animal « que vous n'aimez vraiment pas ». Après un silence (02'), E1 essaye de répondre mais elle a du mal à prononcer le mot "pigeon" si bien que l'enseignant ne le comprend pas (ligne 5). 
fois le malentendu phonétique levé par l'enseignant, il reste le malentendu sémantique d'une autre apprenante E2 qui ne comprend pas le sens du mot 'pigeon' : elle interroge directement son enseignant à la ligne 8 " qu'est-ce que c'est ». A la ligne 10 E4 essaye de lui expliquer le sens du mot 'pigeon' en la regardant tout en faisant un geste iconique : celui d'un oiseau en vol dont les ailes sont symbolisées par les deux mains bougeant de haut en bas. Quant à E3, elle donne le mot dans sa langue maternelle 'bidoulki' à la ligne 13.

L'enseignant intervient pour poser la question en français «qu'est-ce que c'est un pigeon ». E4 réitère son geste pour illustrer le mot 'pigeon' et ainsi désambiguïser sa signification auprès de l'enseignant français natif. L'enseignant regarde E4 en reprenant son geste iconique à la ligne 15 (Image 2) puis donne l'explication en français "c'est comme un aigle mais petit ». Il reprend le geste iconique d'un oiseau qui vole puis ramène ses deux mains pour mimer le mot " petit ».

Nous constatons dans cet exemple que les apprenants utilisent un geste pour expliquer le mot 'pigeon', que ce soit entre eux ou avec l'enseignant. Mais lorsque l'incompréhension d'une apprenante persiste, c'est le lexique coréen qui est utilisé, alors que pour l'expliquer à l'enseignant ils maintiennent le geste iconique. Peut-être se plient-ils à la convention tacite de ne pas s'exprimer en coréen pendant un cours de français, ou alors parce que l'enseignant ne maîtrise pas le coréen, étant en Corée depuis 5 ans ? Ce geste illustratif iconique est repris à son compte par l'enseignant qui l'amende avec un autre geste illustratif qui signifie 'petit' et lui permet de proposer une autre explication du mot 'pigeon'. Ces deux gestes, accompagnés de leur traduction, peuvent être perçus dans leur succession comme des gestes pédagogiques au sens défini par Tellier car ils renseignent sur le sens d'un mot nouveau ou important dans la phrase (2010:35).

Dans cette nouvelle transcription avec l'enseignante A, on peut observer un geste de vérification lexicale exécuté par une apprenante coréenne.

Transcription 3 : «Apéritif »

\begin{tabular}{|l|l|l|}
\hline$(1)$ & $P$ & et l'apéRITIF/ qu'est-ce que c'est/ (regarde les étudiantes) \\
\hline$(2)$ & $\left(07^{\prime}\right)$ & \\
\hline$(3)$ & ES & (regardent le document) \\
\hline$(4)$ & E1 & apéritIF/ \\
\hline$(5)$ & P & (regarde E1) \\
\hline$(6)$ & E2 & XXX (regarde E1) \\
\hline$(7)$ & E1 & XXX \\
\hline$(8)$ & E2 & hum:::hum::: (geste 'boire' avec la main : Image 3) (rire) \\
\hline$(9)$ & P & (imite le geste de E2 : Image 3) (rire) \\
\hline
\end{tabular}




\begin{tabular}{|l|l|l|}
\hline$(10)$ & E2 & après DIner::: \\
\hline$(11)$ & P & après le diNER/= \\
\hline$(12)$ & E2 & =hum::: \\
\hline$(13)$ & P & avant aussi \\
\hline$(14)$ & $(02 ')$ & \\
\hline$(15)$ & E2 & (éclat de rire) \\
\hline$(16)$ & P & (rire) \\
\hline$(17)$ & E2 & avant XXX \\
\hline$(18)$ & P & XXX AVANT le repas XXX et LE SOIR ENCore::: \\
\hline$(19)$ & E2 & (éclat de rire) \\
\hline$(20)$ & P & on est bonjour=ça fait:::ça fait pas mal \\
\hline$(21)$ & & ça fait beaucoup (02') boire AVANT LE REPas (02') le midi ou \\
\hline$(22)$ & & le soir XXX un peu d'alcool comme ça (02') alors \\
\hline$(23)$ & & imaginez maintenant un repas. d'une famille XXX \\
\hline$(24)$ & & un apéritif pendant le repas on boit du vin et \\
\hline$(25)$ & & à la fin on boit ce qu'on appelle DIGESTIF/ pour aider à digérer::: \\
\hline$(26)$ & & déjà on a::: (compte avec ses doigts) la journée::: PAF (rire) \\
\hline$(27)$ & E2 & (rire) \\
\hline
\end{tabular}




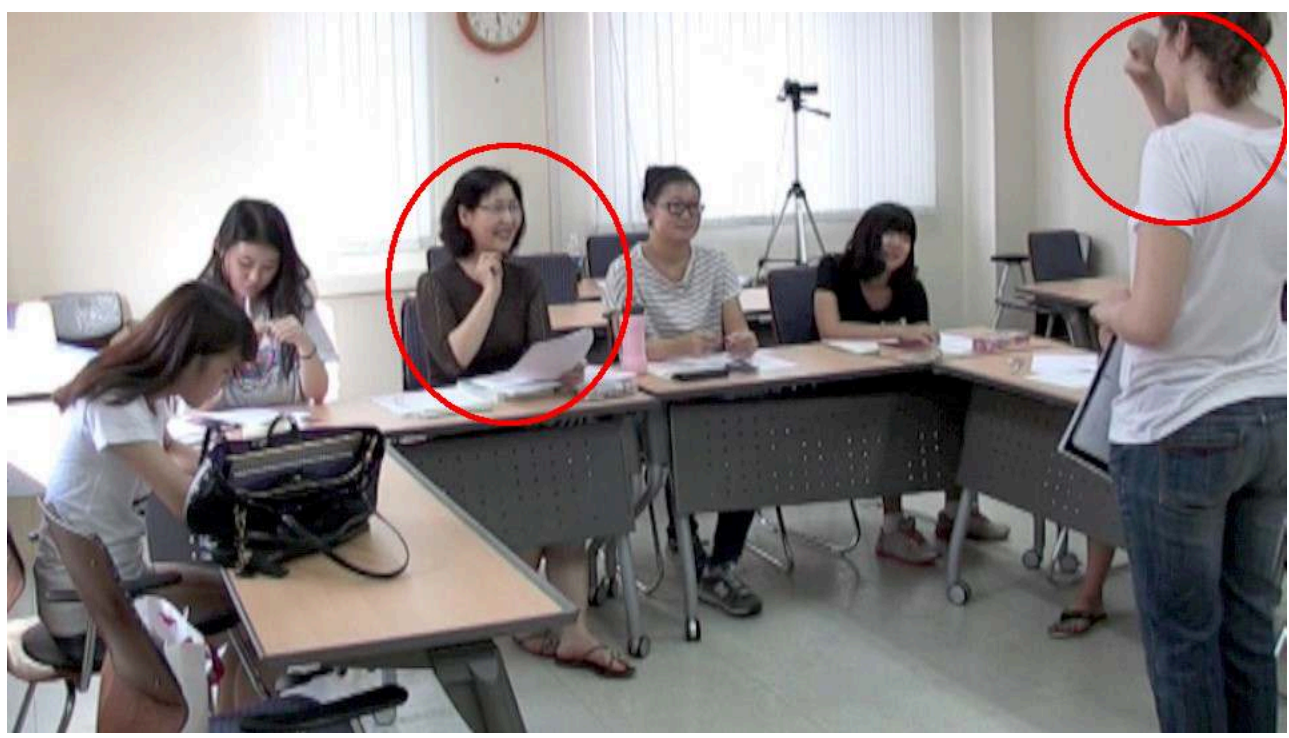

P imite le geste de E2. mot 'boire', on peut mimer simplement la scène de boire en tenant un verre imaginaire dans la main que l'on porte à la bouche. On peut aussi symboliser une bouteille en repliant les doigts et en tendant le pouce : on représente l'action de boire au goulot d'une bouteille. Dans notre corpus (voir l'image 3), l'apprenante coréenne a choisi la première solution, qui représente sans doute la situation de boire un verre d'alcool de riz, car sa main forme un petit arc, comme si elle tenait le petit verre typique utilisé par les Coréens pour boire cet alcool très populaire. L'enseignante a choisi la seconde solution qui illustre la tradition française de boire une bouteille de vin. Dans ce cas précis le référent désigné par le geste n'est pas identique : pour l'apprenante coréenne il s'agit d'un petit verre et pour l'enseignante française d'une bouteille. Les traits saillants caractéristiques du référent sont différents et sont la manifestation d'une différence culturelle.

Comme nous pouvons le voir dans ces transcriptions, les apprenants coréens ont recours aux gestes de vérification lexicale lorsque l'enseignant leur demande la signification d'un lexique émis par l'enseignant lui-même ou qu'il a repris : « Féliciter, qu'est-ce que ça veut dire féliciter?» (exemple 1 - ligne 1), «Qu'est-ce que c'est un pigeon? Vous pouvez nous dire? ? (exemple 2 - ligne 14) et «Et l'apéritif, qu'est-ce que 
c'est?» (exemple 3 - ligne 1). Les gestes de vérification lexicale sont la réponse des apprenants à la requête des enseignants au sujet d'un lexique dont les apprenants sont récepteurs. Ils l'utilisent pour indiquer ce qu'ils savent et éventuellement cherchent à confirmer ou à partager. Ils sont le signal de la compréhension du sens d'un mot ou d'une idée, même si les apprenants ne sont pas capables de l'expliquer en langue cible. Souvent les apprenants se limitent aux gestes de vérification lexicale sans répéter le mot cible et sans l'accompagner d'une explication verbale. Ces gestes pallient une lacune langagière au risque de devenir un mode exclusif de communication. Le geste des apprenants est souvent repris par l'enseignant qui se l'approprie, le modifie ou le complète avec un autre geste, et finalement lui confère ainsi une fonction pédagogique.

\section{4-2. Gestes de compensation lexicale}

33 A la suite à une incompréhension de la part des enseignants lors d'un échange, les apprenants peuvent être tentés de recourir aux gestes afin de désambiguïser un mot qu'ils ont employé, recourant ainsi à une stratégie communicative de compensation (Gullberg, 1998). Plutôt que de se lancer dans une séquence explicative en français qui serait trop difficile à mener à terme, ils préfèrent compenser cette lacune langagière en utilisant un geste illustratif iconique. Nous proposons de nommer ce type de gestes " gestes de compensation lexicale».

Exemple 4 :

Cet extrait a lieu pendant une conversation initiée par l'enseignante $\mathrm{A}$ sur le thème des fêtes d'anniversaire.

Transcription 4 : « Pocketball »

\begin{tabular}{|l|l|l|}
\hline$(1)$ & E1 & XXX Pocketball XX= \\
\hline$(2)$ & P & =pocketball/ j'ne sais pas (est tournée vers E1 et E2) \\
\hline$(3)$ & E2 & oui (geste des mains) \\
\hline$(4)$ & E3 & billard/ pocketball::: \\
\hline$(5)$ & P & (se tourne vers E3) \\
\hline$(6)$ & E2 & pocketBALL (gestes des mains) pocketBALL (regardant P) \\
\hline$(7)$ & E4 & bilia/ (mal prononcé [bija]) \\
\hline$(8)$ & E5 & (geste des mains) \\
\hline$(9)$ & P & AH/BILL:::ard/ (geste des mains : Image 4) ah/ BILLard \\
\hline$(10)$ & $(02 ')$ & \\
\hline$(11)$ & E2 & billard/ \\
\hline$(12)$ & P & (écrit le mot « Billard » sur le tableau) ah::: (soupir) \\
\hline
\end{tabular}




\begin{tabular}{|l|l|l|}
\hline$(13)$ & $\left(04^{\prime}\right)$ & \\
\hline$(14)$ & $P$ & tu m'as dit pocketball/ (en regardant E2) \\
\hline$(15)$ & & je ne comprends pAS/ (geste des mains) (rire) \\
\hline$(16)$ & ES & (rire) \\
\hline$(17)$ & P & XXX (rire) ok::: billard/ \\
\hline$(18)$ & & (regarde le tableau et le désigne avec le doigt) \\
\hline$(19)$ & ES & BILLARD \\
\hline
\end{tabular}

Image 4

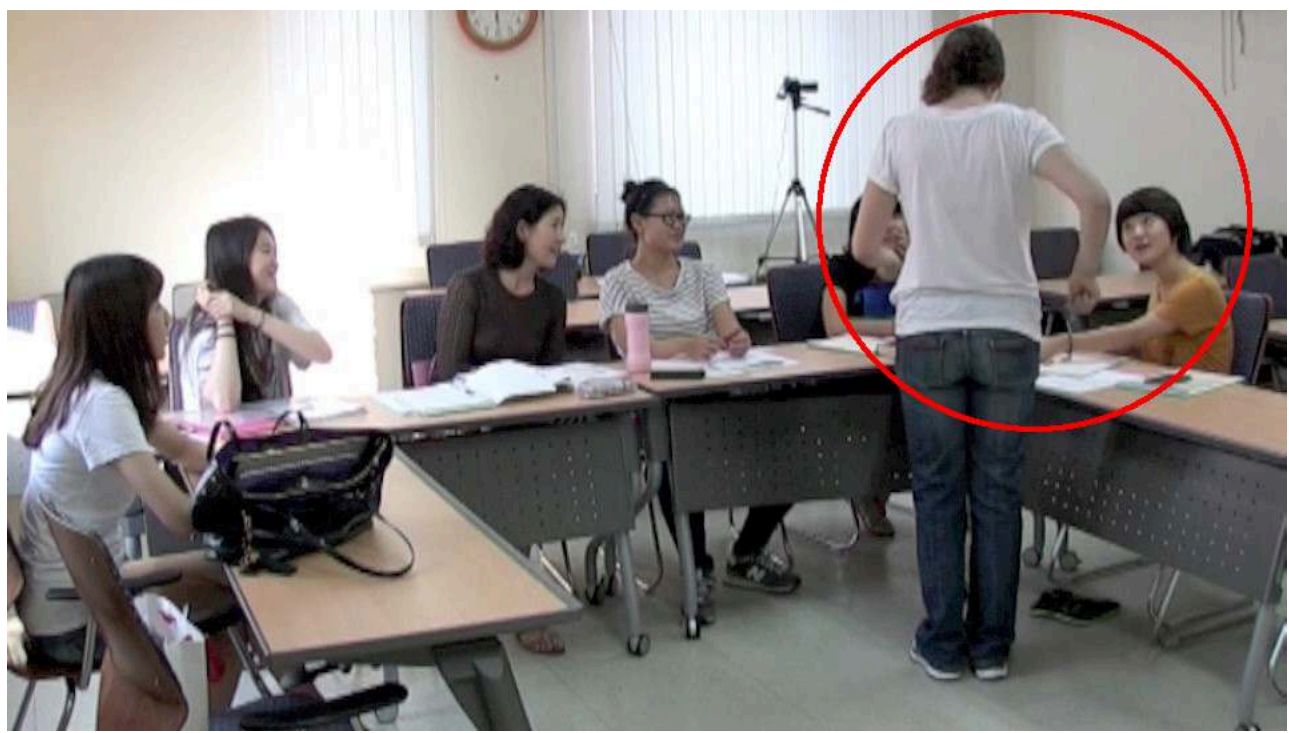

«Ah! Billard! »

L'enseignante A pose la question: "Qu'est-ce que vous faites pendant la fête d'anniversaire? » Les apprenants répondent chacun leur tour.

A la ligne 1, E1 répond 'pocketball'. Mais l'enseignante ne comprend pas ce mot qui se prononce à l'anglaise et elle signale clairement son incompréhension à la ligne 2 «pocketball ? J'ne sais pas ». Une autre apprenante E2 intervient alors pour expliquer ce mot 'pocketball' d'une façon gestuelle : une main repliée vers son ventre et l'autre qui bouge d'avant en arrière dans un geste iconique qui représente l'action de jouer au billard. Voyant que l'enseignante ne comprend toujours pas, E2 réitère son geste de compensation lexicale tout en accentuant la dernière syllabe du mot 'pocketball' «pocketBALL, pocketBALL/» à la ligne 6 en fixant du regard son enseignante. Si les apprenantes insistent tant sur le mot pocketball, c'est sans doute parce qu'elles pensent que l'enseignante connaît ce mot emprunté à l'anglais. Mais le français ne partage pas les mêmes mots anglais que les Coréens et cela génère une incompréhension. Il n'existe pas de nom coréen pour désigner un pocketball alors qu'en français il existe le nom 
billard. Ce lexique exolingue non partagé est peut-être connu par l'enseignante mais il n'est pas reconnu dans le contexte d'un cours de FLE avec des apprenants coréens.

A la ligne 4 une apprenante prononce le mot français correspondant mais l'enseignante ne l'entend peut-être pas, puis à la ligne 7 une autre apprenante essaye à son tour de dire le mot français mais le prononce mal [bija]. Avec ces indices verbaux et non verbaux, l'enseignante finit par comprendre le sens de ce mot exolingue 'pocketball' et reprend le geste de E2 (Image 4) en s'exclamant «ah billard! ». Il est intéressant de remarquer que tous les apprenants regardent le geste de E2. On peut considérer que ce regard collectif des apprenants participe à son soutien: il y a un consensus des apprenants sur le mot énoncé par l'un d'entre eux, une référence commune à tous les apprenants sauf pour l'enseignante. Cette tension voit sa résolution avec l'exclamation de l'enseignante puis le débriefing de la séquence de négociation du sens à partir de la ligne 14 « tu m'as dit pocketball, je ne comprends pas».

On peut remarquer à la ligne 9 que le geste de E2 est réalisé de manière discrète alors que l'enseignante fait un geste plus ample. Signalons que E2 est assise alors que l'enseignante est debout: est-ce la position ou la timidité de l'apprenante qui la pousse à faire un geste étriqué ? Ou bien alors la différence d'amplitude est-elle corrélée à l'adressage du geste: le geste de l'apprenante n'est destiné qu'à l'enseignante sans intention de le rendre visible aux autres qui connaissent le terme pocketball alors que celui de l'enseignante est adressé à toute la classe ? Malgré tout, E2 a essayé de faire comprendre ce mot à l'enseignante en ayant recours à un geste de compensation lexicale qui est venu en complément de l'utilisation d'un lexique anglais affirmé et d'un lexique français murmuré ou mal prononcé.

\section{Exemple 5 :}

41 Voici un autre exemple de lexique exolingue non partagé lors d'un cours de conversation avec l'enseignant $\mathrm{C}$.

Transcription $5:$ : CEO »

\begin{tabular}{|l|l|l|}
\hline$(1)$ & P & pourquoi SI tôt/ \\
\hline$(2)$ & E1 & parce que j'ai::: un cours:::à:::une heure::: comme:::c'est::: \\
\hline$(3)$ & P & oui à une \\
\hline$(4)$ & & heure (fait le chiffre 1 avec le doigt) \\
\hline$(5)$ & E1 & oui NEUF/ (fait le chiffre 9 avec les doigts) \\
\hline$(6)$ & E2 & à neuf heures \\
\hline$(7)$ & P & (rire) à neuf heures à neuf heures ah tu as un cours à neuf heures \\
\hline$(8)$ & & tu es fatigué maintenant/ (regarde sa liste de présence)=un peu \\
\hline$(9)$ & & fatigué/= \\
\hline$(10)$ & E1 & oui \\
\hline
\end{tabular}




\begin{tabular}{|c|c|c|}
\hline (11) & & $=$ oui $=$ \\
\hline (12) & & $=$ oui. pendant trois heures::: (fait le chiffre 3 avec les \\
\hline (13) & & doigts) \\
\hline (14) & $\mathrm{P}$ & un cours de trois heures/= \\
\hline (15) & E1 & $=$ oui $=$ \\
\hline (16) & $\mathrm{P}$ & $=$ un cours de quoi/ \\
\hline (17) & E1 & $e 0:::$ \\
\hline (18) & E2 & $\mathrm{ah}=$ \\
\hline (19) & E1 & $=C E O$. (prononce à la française) $C E O$ (prononce à l'anglaise) \\
\hline (20) & $\mathrm{P}$ & CEO/j'ne comprends pas \\
\hline (21) & E2 & $C E O=$ \\
\hline (22) & E1 & $=C E O$ \\
\hline (23) & $\mathrm{P}$ & mais c'est quoi:::c'est::: \\
\hline (24) & E2 & le directeur ge:::l'entreprise/= \\
\hline (25) & E1 & =che:::chef:::ex:::ex:::pré:::de:::ex:::meoyeossdeora ${ }^{2}$ (parle coréen) \\
\hline (26) & & (rire) \\
\hline (27) & E2 & $e 0^{3} / \mathrm{AH} /$ \\
\hline (28) & & pour devenir=pour devenir:::les directeurs de l'entreprise= \\
\hline (29) & & (geste de battement) \\
\hline (30) & ES & (rire) \\
\hline (31) & $\mathrm{P}$ & $=$ oui $=$ oui $=$ oui $=$ oui \\
\hline (32) & & ah. pour devenir directeur d'entreprise \\
\hline (33) & E2 & étudier (geste avec les doigts : Image 5) \\
\hline (34) & & le:::économie:::eom:::: (geste et mouvement de la tête) \\
\hline (35) & $\mathrm{P}$ & AH:::oui= \\
\hline (36) & E1 & $=\mathrm{ah}$ meoyeossdeora $/=$ (parle coréen) \\
\hline (37) & E2 & $=e \mathrm{om} /$ \\
\hline
\end{tabular}




\begin{tabular}{|l|l|l|}
\hline$(38)$ & P & d'accord $=$ \\
\hline$(39)$ & E3 & =économie \\
\hline$(40)$ & $\left(03^{\prime}\right)$ & \\
\hline$(41)$ & P & d'accord d'accord alors d'accord. Seongmin/ \\
\hline
\end{tabular}

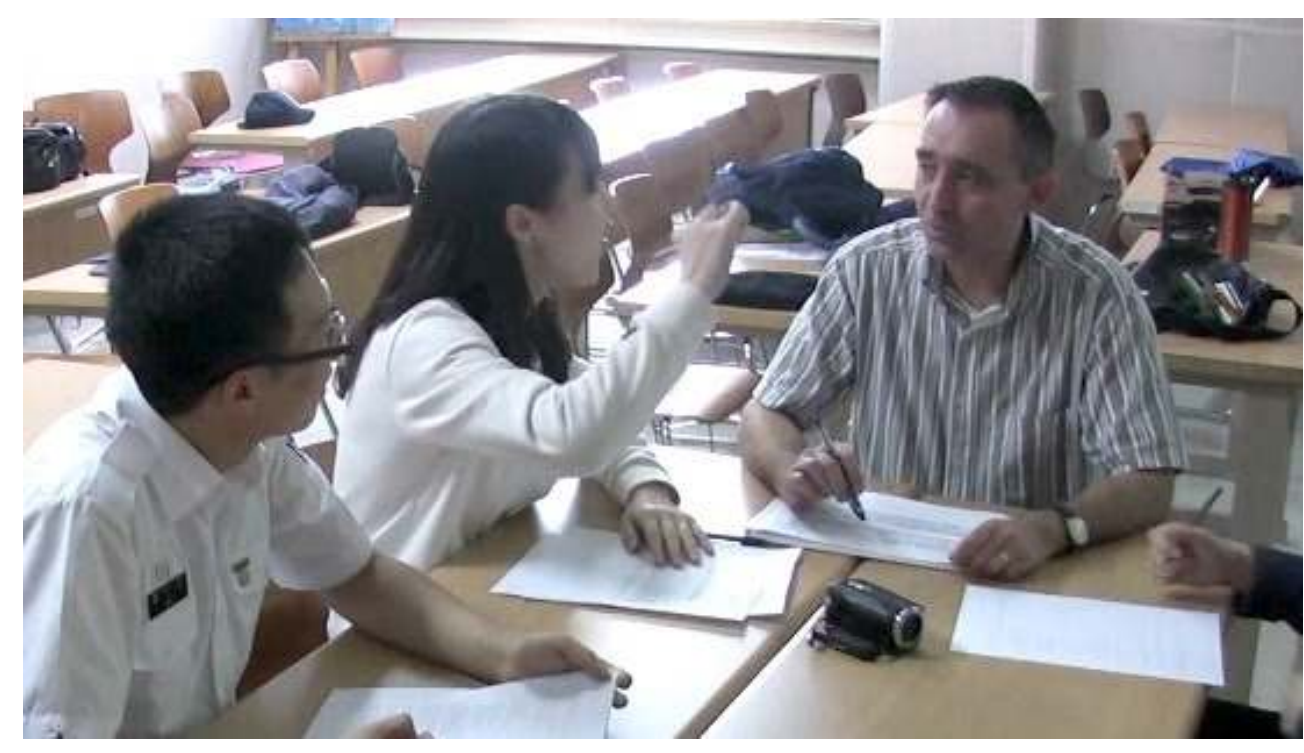

«Etudier... »

Nous allons voir ici comment les apprenants coréens utilisent un geste de compensation lexicale pour expliquer le mot exolingue 'CEO' qui est l'abréviation de Chief Executive Officer. Ce terme anglais est très utilisé en Corée, alors qu'en France on lui préfère le terme de Président, ou plus largement l'acronyme P.D.G. pour Président Directeur Général.

E1 explique la raison de sa fatigue : elle a dû se lever tôt le matin pour assister à un cours de management d'entreprise ; elle prononce alors le mot 'CEO'. Mais l'enseignant ne comprend pas ce mot et le dit clairement à la ligne 20 «CEO, j'ne comprends pas ». E2 intervient volontairement à plusieurs reprises pour aider sa camarade à faire comprendre à l'enseignant le mot exolingue 'CEO' qui est partagé par tous les apprenants coréens. Elle prend la parole à la ligne 27 à la faveur du marqueur discursif coréen «eo/» ('heu'). Ici ce marqueur ne signifie pas son incompréhension mais plutôt qu'elle cherche le moment propice pour intervenir. Elle utilise un geste de battement en répétant la même phrase "pour devenir=pour devenir=les directeurs de l'entreprise » afin d'insister sur l'objectif du cours de management qui vise à enseigner comment devenir directeur d'entreprise, c'est-à-dire 'CEO'. A la ligne 32 l'enseignant reprend ce que E2 a dit mais, même s'il répète plusieurs fois 'oui', il ne semble pas comprendre. E2 enrichit son explication en utilisant le geste de compensation lexicale qui est un geste illustratif iconique et qui signifie "étudier» (Image 5) : elle mime l'action d'écrire. Puis elle complète son argumentation en livrant un deuxième mot clé " économie » qu'elle tente d'illustrer avec un geste qui se voudrait peut-être iconique 
mais qui échoue en geste de battement. Ce n'est qu'à ce moment-là que l'enseignant regarde le geste d'E2 et semble comprendre puisqu'il déclare « Ah oui, d'accord ».

Contrairement aux exemples précédents, le mot 'CEO' est difficile à illustrer avec un geste. Il faut donc que l'explication des étudiants glisse vers une des fonctions d'un directeur, à savoir 'étudier l'économie'. Et finalement c'est seulement le mot 'étudier' qui sera mimée par le geste 'écrire'.

Nous voyons ici qu'il y a une harmonie discursive complète chez l'apprenante E2 :

- utilisation du marqueur discursif « eo » qui marque son engagement dans l'échange ;

- argumentation complètement construite en français: «pour devenir les directeurs de l'entreprise »;

- utilisation du geste communicatif de battement ;

- illustration du discours avec un geste de compensation lexicale en transposant le mot 'CEO' vers « étudier l'économie ».

Remarquons qu'à deux reprises (lignes 25 et 36) E1, n'arrivant pas à donner le nom en français, s'adresse à E2 en coréen en lui disant «meoyeossdeora» que l'on peut interpréter par « comment dit-on?». Mais elle ne cède pas à la facilité de donner une explication en coréen. Estime-t-elle que l'enseignant ne le comprendrait pas, ou respecte-elle la consigne de ne pas s'exprimer en coréen?

\section{Exemple 6 :}

Cet exemple est extrait d'un corpus adjacent qui se situe en France dans un cours de FLE à l'université Lyon 2. Les étudiants sont de multiples nationalités et parmi eux se trouve une apprenante coréenne.

Transcription 6 : « Hoho »

\begin{tabular}{|l|l|l|}
\hline$(1)$ & P & ok et:::le tague:::hoho c'est quoi heu::: (03') \\
\hline$(2)$ & & Kyo (silence 03') hoho c'est quoi/ \\
\hline$(3)$ & EC & HOHO \\
\hline$(4)$ & P & HOHO oui (avec la tête) (sourire) \\
\hline$(5)$ & E1 & c'est quoi:::(rire) \\
\hline$(6)$ & ES & (rire) \\
\hline$(7)$ & P & alors oui (désigne le tableau) qu'est-ce que ça veut dire/ (sourire) \\
\hline$(8)$ & EC & [(02') RIen:::hi/ \\
\hline$(9)$ & E2 & [quelque chose de quand même::: \\
\hline$(10)$ & EC & HOHO (geste avec les bras : Images 6 et 7) \\
\hline$(11)$ & E1 & comment/ \\
\hline$(12)$ & P & CHOuette (regarde les élèves) ça veut dire cHOuette/ \\
\hline
\end{tabular}




\begin{tabular}{|l|l|l|}
\hline$(13)$ & & (geste avec les bras : Images 8 et 9) \\
\hline$(14)$ & E2 & oui oui chouette \\
\hline$(15)$ & P & ok ok \\
\hline$(16)$ & EC & oui::: (rire) \\
\hline$(17)$ & P & bon:::alors les voilà \\
\hline
\end{tabular}

Image 6

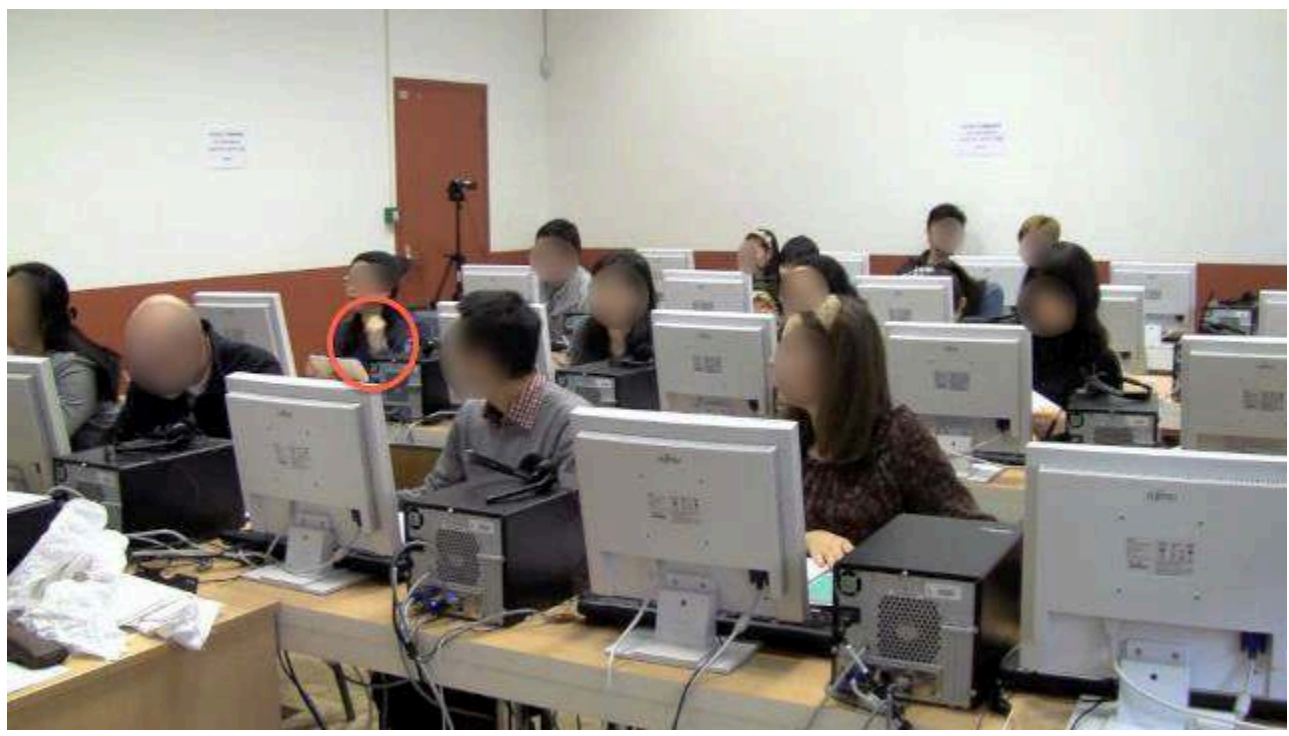

Image 7

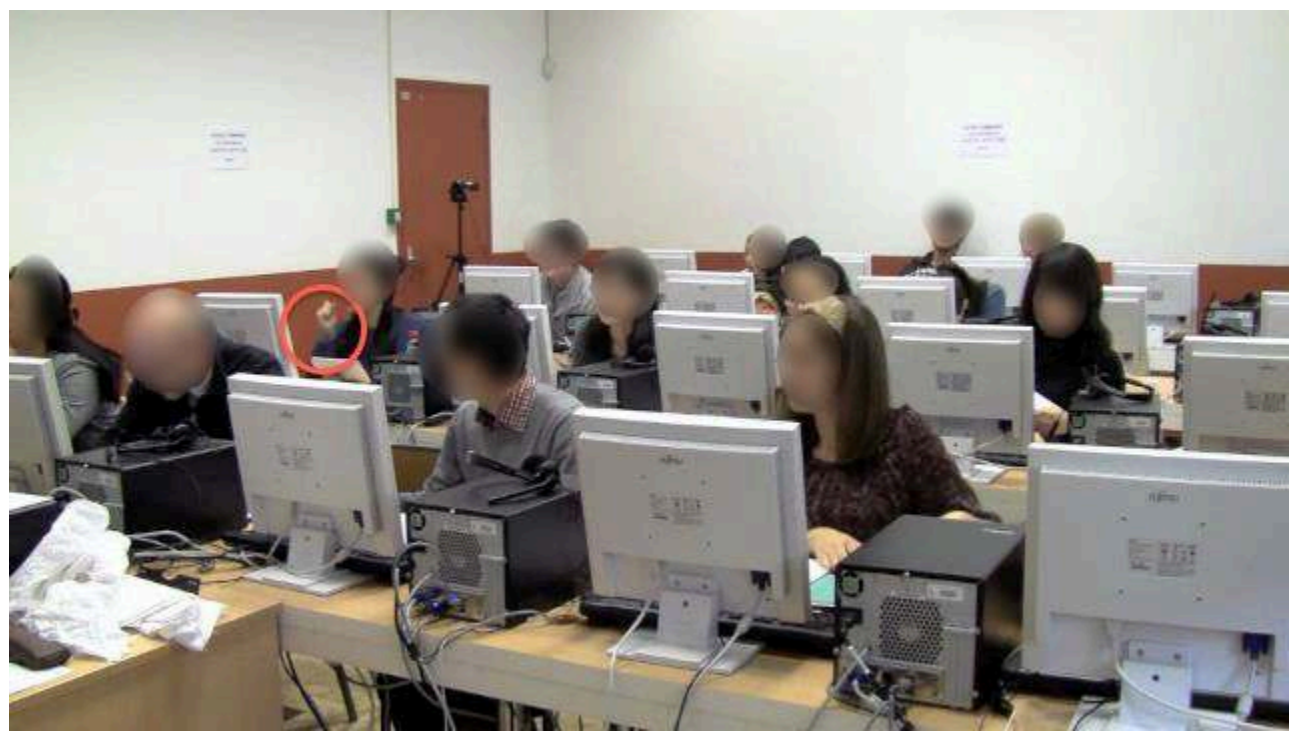

« Hoho! » (images 6 et 7 ) 
Image 8

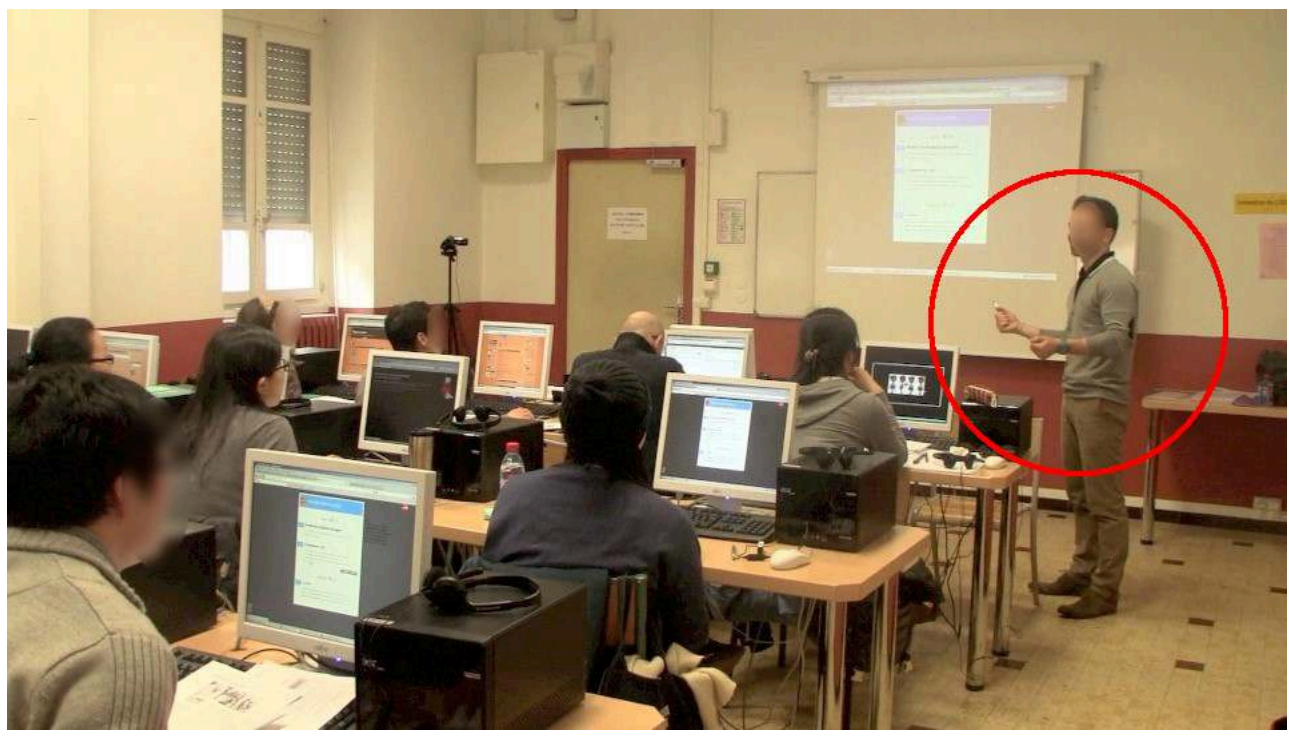

Image 9

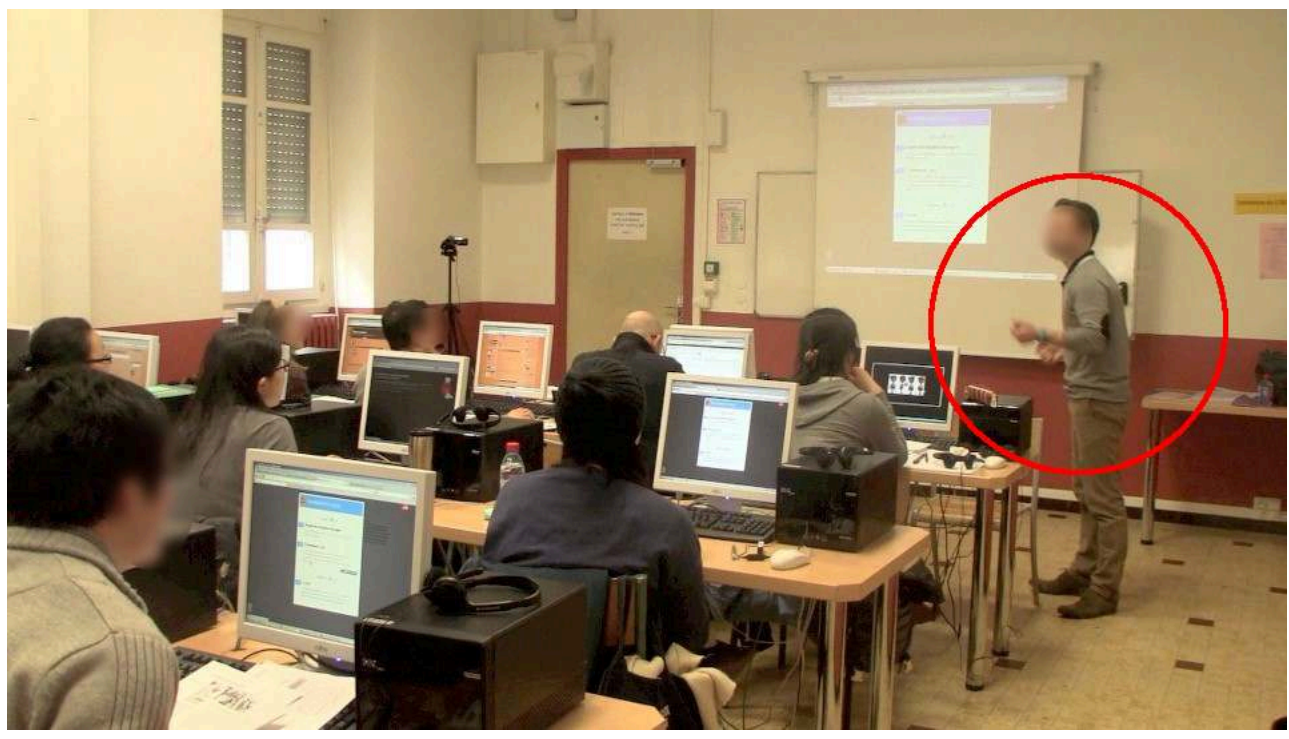

«Chouette! Ça veut dire chouette! » (images 8 et 9)

49 Il s'agit pour l'apprenante coréenne (EC) de faire comprendre l'interjection culturelle spécifique à la Corée 'hoho', qui est l'onomatopée du rire.

50 A la ligne 1, l'enseignant D corrige le travail rendu par EC. Il y remarque un symbole associé à une interjection exotique 'hoho' et lui en demande la signification " hoho c'est quoi heu... », puis il réitère sa question " qu'est-ce que ça veut dire ? » à la ligne 7. EC commence par répondre « rien:::hi/ » car elle n'arrive pas à fournir une explication. E2 intervient pour l'aider mais elle ne finit pas sa phrase.

51 EC fait alors un geste abstrait avec les bras (Images 6 et 7) : elle les lève et les bouge alternativement de haut en bas comme si elle courait; est-ce un geste illustratif qui mime la course ? Mais ce geste accompagne le mot « hoho » en le cadençant; est-ce un geste de battement coverbal ? Ou bien est-ce un geste emblème quasi-linguistique qui 
peut se substituer à l'interjection? A vrai dire il est difficile de le savoir, mais nous pouvons supposer qu'il s'agit d'un geste de compensation lexicale.

Apparemment l'enseignant ne semble pas vraiment saisir le sens de ce mot et du geste qui l'accompagne. Le geste de compensation lexicale de l'apprenante coréenne n'est pas efficace pour en faire comprendre le sens. En effet l'enseignant propose la traduction «chouette/ ça veut dire chouette/» en faisant un geste avec ses bras à la ligne 13 (Images 8 et 9). Cette incompréhension culturelle n'a pas pu être complètement levée par le geste. La signification du geste n'étant pas pleinement comprise, la reproduction du geste par l'enseignant n'est pas correcte: l'apprenante coréenne utilise les deux bras et les bouge comme si elle courait, ses bras se meuvent verticalement; l'enseignant utilise aussi les deux bras mais les laisse presque immobiles à l'horizontale. 'Hoho' est un mot mimétique pour exprimer le rire en coréen et il révèle l'importance des composantes orales de la langue coréenne. Mais nous pouvons penser que, plus que le geste, c'est l'intention portée par l'apprenante coréenne qui apporte un sens et que c'est cette intention qui a orienté l'enseignant dans le choix de la traduction. En effet dans cette séquence l'apprenante parle fort, avec entrain, fait des gestes amples et se positionne clairement dans un registre joyeux.

Comme nous pouvons le voir dans ces transcriptions, les apprenants coréens ont recours aux gestes de compensation lexicale lorsque l'enseignant exprime clairement son incompréhension d'un mot émis par les apprenants : "pocketball ? J'ne sais pas » (exemple 4 - ligne 2), «CEO, j'ne comprends pas » (exemple 5 - ligne 20) et « hoho c'est quoi heu... » puis «qu'est-ce que ça veut dire? » (exemple 6 - lignes 1 puis 7). Pour résoudre les incompréhensions, il aurait fallu donner le mot exact en français : 'billard', 'président' et 'rire', mais apparemment les apprenants ne les connaissent pas ou les prononcent mal. Les gestes de compensation lexicale sont la réponse des apprenants à la requête des enseignants au sujet d'un lexique dont les apprenants sont émetteurs. Ils tentent de résoudre une problématique d'incompréhension des enseignants français natifs à propos de mots exolingues qui sont usités dans le pays de la langue source mais pas partagés par le pays de la langue cible. Ils ne permettent pas toujours de lever intégralement le problème de communication (voir les exemples 5 et 6 ). Ils complètent l'énoncé en utilisant un langage gestuel imagé qui se veut universel. Mais nous avons constaté que certains gestes ont une dimension socioculturelle et qu'ils ne peuvent pas, par conséquent, se prévaloir de l'universalité.

\section{4-3. Geste déictique}

Nous avons vu des gestes iconiques, et même un emblème, utilisés par les apprenants pour illustrer le lexique. Nous n'avons pas pu identifier de gestes métaphoriques. Nous allons analyser la troisième catégorie des gestes coverbaux illustratifs, et qui sont aussi les plus nombreux dans notre corpus : les gestes déictiques. Mais nous ne proposerons pas d'analyses sur les gestes déictiques concrets qui n'ont qu'une simple fonction de pointage et qui dans la stratégie communicative des apprenants peuvent s'apparenter à une recherche de lexique. Nous évoquerons cependant deux exemples de gestes déictiques, certes concrets, mais dont la référence va au-delà de ce qui est pointé.

\section{Exemple 7 :}

Dans ce nouvel extrait, l'enseignante A poursuit le cours de conversation sur le thème des fêtes d'anniversaire en posant à chaque apprenant des questions sur leur manière 
de les fêter. Afin d'établir de la part des apprenants une amorce de co-énonciation, l'enseignante pose beaucoup de questions adaptées du contenu du cours. Ce mode conversationnel pousse les apprenants à pratiquer le français et induit généralement une réponse illustrée par des gestes, et bien souvent des pointages avec la main ou le doigt.

Transcription 7 : « Petite sœur »

\begin{tabular}{|l|l|l|}
\hline$(1)$ & P & elle a quel âGE/ \\
\hline$(2)$ & E1 & eum $^{5}::$ (désigne une étudiante avec le doigt : Image 10) \\
\hline$(3)$ & ES & $(02$ ') (rire) \\
\hline$(4)$ & P & (rit et regarde l'étudiante désignée par E1) (imite la geste de E1) \\
\hline$(5)$ & E1 & (rire) EO/ myeotch sariji6/ (en coréen) aH::: dix-sept:::ANS::: \\
\hline$(6)$ & P & le même âge le MEME AGE que Suji ou::: pareil que Suji \\
\hline$(7)$ & & le même âge d'accord le MEME AGE/ \\
\hline$(8)$ & E1 & eum (fait oui avec la tête) \\
\hline$(9)$ & P & (écrit le mot sur le tableau) \\
\hline
\end{tabular}

Image 10

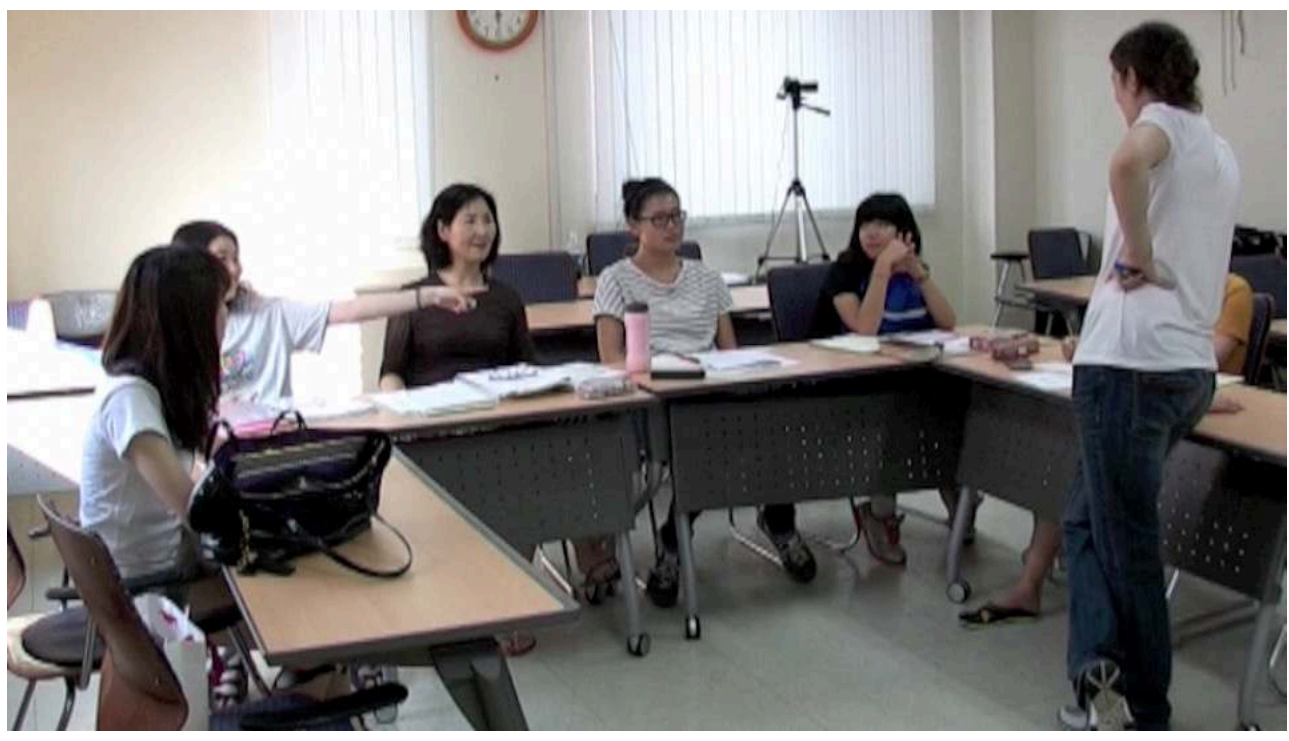

« eum... »

57 Cet extrait nous permet d'analyser un geste déictique appuyé. L'apprenante E1 y narre la fête d'anniversaire de sa sœur, et l'enseignante lui demande alors son âge. Après une hésitation marquée par le marqueur discursif « eum » à la ligne 2, E1 pointe son index vers une camarade de la classe qui se prénomme Suji (Image 10). Ce geste a un contenu 
implicite qui est 'ma petite sœur a le même âge que Suji'. Pendant tout son discours l'étudiante reste le doigt pointé vers sa camarade et ne quitte pas l'enseignante des yeux : ce geste insistant se substitue même à son discours et constitue un élément de réponse.

Quant à l'enseignante, son regard est d'abord fixé sur E1 et, après le geste de pointage de E1, elle regarde enfin la personne désignée et reproduit le même geste que E1, signifiant ainsi qu'elle a compris le contenu implicite. L'enseignante en profite alors pour livrer la formulation correcte de la notion grammaticale de comparaison «le même âge que » puis l'écrit au tableau.

Un peu plus loin dans cet échange, on s'aperçoit que l'apprenante connaît les chiffres en français puisqu'elle les traduit en auto-reformulation ('ah::: dix-sept:::ans'). Elle a utilisé stratégiquement un geste déictique chargé d'un énoncé implicite 'ma sœur a le même âge que Suji' afin de préparer et assurer sa réponse un peu tardive. Elle semble se concentrer essentiellement sur sa recherche de formulation - elle sait dire les chiffres en français - comblant son hésitation et la pause (02) par un geste déictique.

\section{Exemple 8 :}

61 Nous avons rencontré d'autres cas d'utilisation de gestes déictiques remplaçant ou complétant un énoncé partiel, comme dans l'exemple suivant avec l'enseignant B.

Transcription 8 : « Mariage »

\begin{tabular}{|l|l|l|}
\hline$(1)$ & E1 & eo:::mériage mariage imprudent \\
\hline$(2)$ & ES & (rire) \\
\hline$(3)$ & P & d'accord XXX \\
\hline$(4)$ & ES & (rire) \\
\hline$(5)$ & E2 & le mariage imprudent \\
\hline$(6)$ & P & mariage imprudent (il s'approche de E1) (03') \\
\hline$(7)$ & & ah:::tu veux dire::: \\
\hline$(8)$ & E1 & (fait un geste déictique avec la main vers P : Image 11) \\
\hline$(9)$ & P & (rire) tu veux [dire:::XXXX \\
\hline$(10)$ & E1 & [compliment/ XXX (elle éclate de rire et tape dans les mains) \\
\hline$(11)$ & P & (sourire) tu veux dire:::XXX trop vite c'est ça/ \\
\hline$(12)$ & ES & oui oui (rire) \\
\hline
\end{tabular}




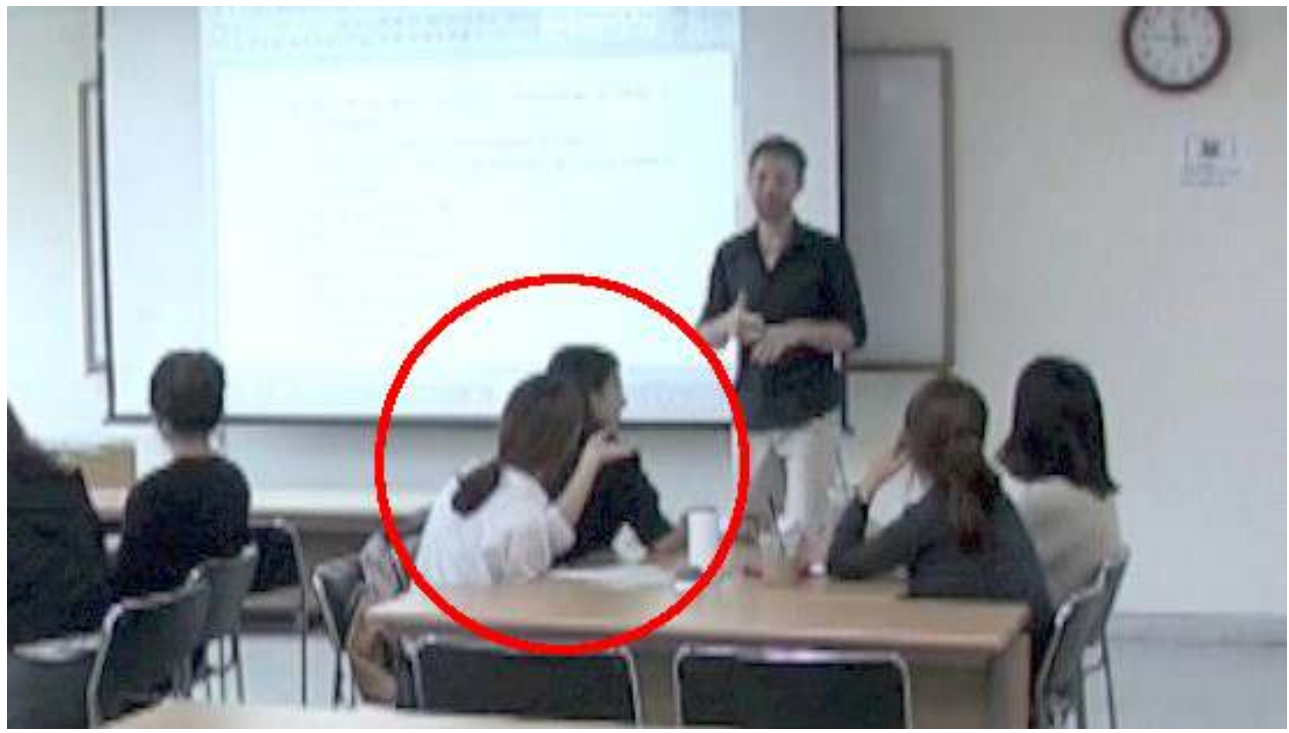

« Le mariage imprudent. »

$\mathrm{Au}$ cours d'un débat sur le mariage, l'apprenante E1 semble vouloir dire que l'enseignant a lui-même contracté un mariage imprudent car il se serait marié jeune. $\mathrm{Au}$ lieu de formuler la phrase «Tu as fait un mariage imprudent» ou "Tu as été imprudent parce que tu t'es marié jeune », elle dit "le mariage imprudent » qu'elle complète par un geste de la main désignant l'enseignant (Image 11). Elle insiste sur ce geste en le maintenant assez longtemps à tel point qu'il se substitue à sa parole. Les rires des apprenants et de l'enseignant indiquent qu'ils ont compris le message incomplet délivré par E1. Plus particulièrement, l'enseignant confirme qu'il a compris en reformulant le message ainsi : «Tu veux dire 'trop vite', c'est ça?». Nous pouvons remarquer que le geste déictique de l'apprenante n'est pas un doigt pointé, ce qui pourrait être un manque de respect envers un enseignant, mais plutôt une désignation par la main, presque comme une invitation à compléter sa phrase.

On peut comprendre que le niveau de français des apprenants coréens ne leur permet pas d'avoir de riches interactions verbales avec leurs enseignants. Dans ces deux exemples, les gestes déictiques ne servent pas à illustrer un lexique mais plutôt à exprimer une phrase ou une notion grammaticale complexe. Ils montrent que l'énoncé implicite du geste déictique se substitue dans une certaine mesure au langage verbal.

\section{Conclusion}

L'analyse des gestes permet de mieux comprendre les interactions en classe de langue entre enseignants et apprenants. Dans cette étude nous nous sommes spécifiquement intéressés aux gestes des apprenants coréens, ce qui n'avait pas encore fait l'objet de recherches jusqu'à maintenant.

Notre étude révèle une prépondérance des gestes illustratifs dans un contexte d'apprentissage du français. Cette prépondérance s'explique par la nécessité de reformuler, d'expliciter le contenu des énoncés, voire de pallier une lacune langagière. Nous avons identifié des gestes exécutés apparemment intentionnellement dans le but d'illustrer le discours verbal (applaudir, pigeon, boire, billard, étudier, rire). 
Majoritairement iconiques, ces gestes de vérification lexicale et de compensation lexicale ont révélé des spécificités socioculturelles. Ces particularités sont apparues à l'occasion d'un lexique exolingue non partagé (Pocketball, CEO) ou de gestes marqués culturellement (petit verre d'alcool, interjection Hoho). Par ailleurs, si le corpus présente de nombreux gestes déictiques qui assurent la fonction primaire de désignation, nous en avons identifié deux qui semblent porteurs de plus de sens. En effet ils se substituent à une forme verbale qui serait complexe à produire en langue cible (« Ma sœur a le même âge que Suji », « Tu as fait un mariage imprudent »). On ne peut plus alors les considérer comme de simples gestes de pointage. Tous les gestes coverbaux illustratifs des apprenants coréens semblent donc s'inscrire dans une stratégie communicative pour surmonter des problèmes discursifs et grammaticaux comme le suppose Gullberg $(1998,2005)$. Et cela leur permet aussi de respecter le contrat tacite qui bannit l'utilisation de la langue maternelle dans la salle de classe pendant un cours de langue étrangère.

Nous nous sommes intéressés uniquement aux gestes coverbaux illustratifs des apprenants. Mais les apprenants coréens ont une mimogestualité riche. Les fréquents hochements de tête, gestes synchronisateurs régulateurs du discours, servent aux apprenants à signifier leur participation au cours. D'autres éléments non verbaux mériteraient également une analyse, comme le regard, dont la fonction régulatrice semble constituer une stratégie d'évitement (quand l'apprenant fixe sa feuille en tentant de fuir le regard de l'enseignant par exemple). S'ils sont parfois révélateurs d'une culture, les gestes effectués par les apprenants sont généralement repris à leur compte par les enseignants et adaptés à leur propre représentation du monde, comme une reformulation de ce qui est dit.

\section{BIBLIOGRAPHIE}

Azaoui, B. (2014a) Multimodalité des signes et enjeux énonciatifs en classe de FL1/FLS, In M. Tellier \& L. Cadet (dir.), Le corps et la voix de l'enseignant : mise en contexte théorique et pratique, Paris: Editions Maison des Langues, p. 115-126.

Azaoui, B. (2014b) Très Bien, super, génial et Cie. Impact du contexte classe sur les félicitations multimodales de l'enseignant, In Aguilar, J ; Brudermann, C. \& M. Leclère (dir.), Langues, cultures et pratiques en contexte : interrogations didactiques, Paris: Riveneuve éditions, p. 47-74.

Calbris, G. \& L. Porcher (1989) Geste et communication, Paris: Didier.

Calbris, G. (2011) Elements of Meaning in Gesture, Amsterdam: John Benjamins Publishing, Colletta, J.-M. (2004) Le développement de la parole chez l'enfant âgé de 6 à 11 ans, Liège: Mardaga. Colletta, J.-M. (2005) Communication non verbale et parole multimodale : quelles implications didactiques ?, Recherches et applications - Le français dans le monde, 38, Paris: CLE international, p. $32-41$. 
Colletta, J.-M. (2011) Le co-développement du langage et des gestes chez l'enfant âgé de trois ans et plus - avancées récentes, Rééducation orthophonique, 246, p. 59-71.

Cosnier, J. (1982) Communications et langages gestuels, In Cosnier, J., Coulon, J., Berrendonner, A. \& C. Kerbrat-Orecchioni (dir.), Les voies du langage : communications verbales gestuelles et animales, Paris: Dunod, p. 255-304.

Cosnier, J. (1997) en collab. avec Vaysse, J., Sémiotique des gestes communicatifs, Nouveaux actes sémiotiques, 52, p. 7-28.

Dejean-Thircuir, C. \& F. Mangenot (2006) Les échanges en ligne dans l'apprentissage et la formation, Cadrage et présentation, In Dejean-Thircuir, C. \& F. Mangenot (coord.) Les échanges en ligne dans l'apprentissage et la formation, Recherches et applications - Le français dans le monde, 40, Paris: CLE International, p. 5-13.

Gagné, G., Sprenger-Charolles L., Lazure, R. \& F. Rope (1989) Recherches en didactique et acquisition du français langue maternelle (1970-1984), Tome 1 : Cadre conceptuel, thésaurus et lexique des mots-clés, Bruxelles, Paris, Montréal: De Boeck-Université, Institut National de la Recherche Pédagogique, p. 39-61.

Gullberg, M. (1998) Gesture as a Communication Strategy in Second Language Discourse: A Study of Learners of French and Swedish, Lund: Lund University Press.

Gullberg, M. (2005) L'expression orale et gestuelle de la cohésion dans le discours de locuteurs langue 2 débutants, Acquisition et interaction en langue étrangère, 23, p. 153-172.

Kendon, A. (2004) Gesture: visible action as utterance, Cambridge: Cambridge University Press.

McCafferty, S. \& Ahmed, A. (2000) The appropriation of gestures of the abstract by L2 learners? In J. P. Lantolf (ed.), Sociocultural theory and second language learning, p. 199-218

McNeill, D. (1992) Hand and Mind: What Gestures reveal about thought, Chicago: The University of Chicago Press.

McNeill, D. (2005) Gesture \& thought, Chicago: The University of Chicago Press.

Pavelin, B. (2002) Le geste à la parole, Toulouse: Presses Universitaires du Mirail.

Stam, G. (2008) What Gestures Reveal About Second Language Acquisition, In McCafferty, S. and Stam, G., Gesture: Second Language Acquisition and Classroom Research, Mahwah, NJ: Lawrence Erlbaum Publishers.

Tellier, M. (2008) Dire avec des gestes, In Chnane-Davin, F. \& J.-P. Cuq (Eds) Du discours de l'enseignant aux pratiques de l'apprenant en classe de français langue étrangère, seconde et maternelle, Recherches et Applications - Le Français dans le monde, 44, Paris: CLE International, p. 40-50.

Tellier, M. (2010) Faire un geste pour l'apprentissage : Le geste pédagogique dans l'enseignement précoce, In Corblin, C. \& J. Sauvage (Eds) L'apprentissage et l'enseignement des langues vivantes à l'école, Impacts sur le développement de la langue maternelle, Paris: L'Harmattan (Enfance \& Langages), p. 31-54.

Traverso, V. (1999) L'analyse des conversations, Paris: Nathan.

Traverso, V. (2008) Analyser un corpus de langue parlée en interaction : questions méthodologiques, Verbum, 30 (4), p. 313-328. 


\section{ANNEXES}

\section{Convention de transcription}

\begin{tabular}{|l|l|}
\hline$:::$ & allongement vocalique \\
\hline Bidoulki & mot étranger en italique \\
\hline$\left(09^{\prime}\right)$ & pause chronométrée \\
\hline$\cdot$ & pause non chronométrée \\
\hline / & intonation montante \\
\hline ( ) & notation des gestes \\
\hline [ & chevauchement \\
\hline = & enchaînement rapide \\
\hline exTRA & segment accentué \\
\hline XXX & segment inaudible \\
\hline P & enseignant \\
\hline E1, E2, E3, E4, E5, E6 & apprenant \\
\hline ES & apprenants \\
\hline EC & apprenante coréenne \\
\hline
\end{tabular}

\section{NOTES}

\section{1. « pigeon"}

2. «Comment dit-on?»

3. « heu »

4. « $\mathrm{hm} »$

5. « hm»

6. «Quel âge elle a?»

\section{RÉSUMÉS}

L'objectif de cette contribution est d'identifier et analyser les gestes utilisés par les apprenants coréens face à leur enseignant français dans le cadre de cours de FLE à l'université en Corée du 
Sud. Pour cette analyse de pratiques d'apprenants, nous utiliserons principalement la catégorisation fonctionnelle du geste établie par Cosnier (1982, 1997), dans laquelle nous retiendrons les gestes communicatifs coverbaux illustratifs. Nous évoquerons par ailleurs les dimensions définies par McNeill (1992, 2005). Nous nous pencherons essentiellement sur les nombreux gestes illustratifs, principalement iconiques et déictiques, révélateurs des spécificités discursives et culturelles des apprenants coréens dans leurs interactions avec les enseignants français. Nous tenterons de voir comment le corps des participants à l'échange coconstruit le discours et le sens dans cette situation propice aux malentendus et incompréhensions. Pour cela nous analyserons à chaque fois la réaction de l'enseignant afin de voir si l'intention de l'apprenant a été comprise et la façon dont il a géré l'échange.

The purpose of this contribution is to identify and analyze the gestures used by Korean learners addressed to their French teacher in the context of French as a foreign language courses at the university in South Korea. For this analysis of learner practices, we will use the functional categorization of gestures established by Cosnier $(1982,1997)$, in which we will retain the communicative co-verbal illustrative gestures. We will also recall the dimensions defined by McNeill (1992, 2005). We'll focus on the many illustrative gestures, mainly iconic and deictic, revealing the discursive and cultural specificities of Korean learners in their interactions with French teachers. We have tried to see how the body of participants in the exchange co-constructs discourse and meaning in this situation, which may lead to misunderstanding and incomprehension. For that purpose, we analyzed for each example, the teacher's reaction to the learner's gesture to see if the intention of the learner was understood and the way he managed the exchange.

Our study reveals a preponderance of illustrative gestures in the context of learning French, which can be explained by the need to rephrase, to clarify the contain of the statements, or even to overcome a language gap. We have identified apparently intentional gestures which were performed to illustrate the verbal discourse (applaudir, pigeon, boire, billard, étudier, rire). Mostly iconic, these gestures of lexical verification and lexical compensation revealed sociocultural specificities. These peculiarities appeared on with the use of an exolingual lexicon which is not shared (Pocketball, CEO) or culturally influenced gestures (small glass of alcohol, interjection Hoho). Furthermore, throughout the corpus we have identified many deictic gestures that provide the primary function of designation. But sometimes these gestures go further because they replace a verbal form that would be complex to produce in the target language ("My sister is the same age as Suji", "You made an imprudent marriage"). They can no longer be considered as mere pointing gestures.

All the illustrative co-verbal gestures of Korean learners thus seem to be part of a communicative strategy to overcome discursive and grammatical problems, as Gullberg (2005) pointed out. And it also allows them to respect the tacit contract banning the use of the native language in the classroom during a foreign language course. If they are sometimes indicative of a culture, the gestures performed by the learners are usually taken over by the teachers and adapted to their own representation of the world, as a reformulation of what is being said.

\section{INDEX}

Mots-clés : geste illustratif, geste coverbal, interaction pédagogique, classe de langue

Keywords : illustrative gesture, co-verbal gesture, pedagogical interaction, language class 
AUTEUR

\section{SHIN-TAE KANG}

Institut National des Langues et Civilisations Orientales (INALCO)

Interactions, Corpus, Apprentissages, Représentations (ICAR) UMR 5191

gsht22@gmail.com 